\title{
Immunohistochemical Analysis of Ki-67, p53, p21, and p27 in Benign and Malignant Apocrine Lesions of the Breast: Its Correlation to Histologic Findings in 43 Cases
}

Takuya Moriya, M.D., Kazuhiro Sakamoto, M.D., Hironobu Sasano, M.D., Miwa Kawanaka, M.D., Hiroshi Sonoo, M.D., Toshiaki Manabe, M.D., Jishu Ito, M.D.

Department of Pathology, Tohoku University Hospital (TMo, HS), and Department of Pathology, Kawasaki Hospital, Kawasaki Medical School (KS, MK, JI), Okayama; and Departments of Surgery (HS) and Pathology (TMa), Kawasaki Medical School, Kurashiki, Japan

We examined Ki-67, p53, p21, and p27 immunolocalization in 43 cases of apocrine lesions of the breast and correlated these findings with histologic parameters to understand their biologic significance. Twenty cases were benign, 1 case was borderline, and 22 cases were diagnosed as malignant, including 9 intraductal and 13 invasive apocrine carcinomas. Both the ratio of Ki-67-positive cases (17 of 21 [88.9\%] versus 1 of 19 $[5.3 \%] ; P<.001)$ and the Ki-67 labeling index of positive cases examined $(15.0 \%$ versus $2.7 \%$; $P<.005)$ were significantly higher in malignant than in benign apocrine lesions. None of the benign or borderline cases was immunohistochemically positive for $\mathrm{p53}$, but 15 of 22 malignant cases $(68.2 \%)$ demonstrated p53 $(P<.001)$. In addition, the ratio of p53-positive cases was significantly higher in high nuclear grade cases (11 of $13[84.6 \%]$ ) than in intermediate nuclear grade cases ( 4 of 9 [44.4\%]; $P<.05$ ). P53 immunoreactivity was also positively correlated with the nuclear grade of carcinoma cases examined in this study. Neither p21 nor p27 demonstrated any correlation with histologic parameters or findings of the apocrine lesions. Results of these studies suggest that Ki-67 and p53 may be good markers for differentiation between benign and malignant breast apocrine lesions.

KEY WORDS: Apocrine carcinoma, Apocrine metaplasia, Breast, Immunohistochemistry, Ki-67, p53.

Mod Pathol 2000;13(1):13-18

Copyright () 2000 by The United States and Canadian Academy of Pathology, Inc.

VOL. 13, NO. 1, P. 13, 2000 Printed in the U.S.A.

Date of acceptance: July 22, 1999.

Address reprint requests to: Takuya Moriya, M.D., Department of Pathology, Tohoku University Hospital, 1-1, Seiryo-machi, Aoba-ku, Sendai 980-8574, Japan; e-mail: moriya@patholo2.med.tohoku.ac.jp; fax: 81-22273-5976.
A recent development in noninvasive diagnostic techniques for breast diseases, especially mammography, has contributed greatly to the detection of minute foci of intraductal carcinoma, including cases with diffuse apocrine cytology (1-3). Apocrine carcinoma of the breast has been relatively well characterized, but the clinicopathologic significance of apocrine adenosis (4), atypical apocrine adenosis (5), papillary apocrine change (6), and atypical apocrine hyperplasia (7) has not been characterized in detail.

The presence of focal apocrine metaplasia within epithelial proliferations of the breast is generally considered an important indicator in the histopathologic diagnosis of benignity (8). However, histologically, it is occasionally difficult to interpret the malignant potential of intraductal lesions with diffuse apocrine features, because benign apocrine metaplastic cells may be associated with various degrees of nuclear atypia or the presence of macronucleoli (8). Therefore, we studied various apocrine breast lesions using immunohistochemistry for Ki$67, \mathrm{p} 53, \mathrm{p} 21$, and p27 to examine the biologic significance of these markers in proliferative apocrine lesions of the human breast, especially in relation to the identification of a possible malignant phenotype.

\section{MATERIALS AND METHODS}

All of the materials examined in this study were retrieved from the surgical pathology files of the Department of Pathology, Kawasaki Medical School Hospital, between 1982 and 1997 and from the consultation case files of one of the authors (TMo) between 1994 and 1997. A total of 43 breast lesion cases with diffuse or extensive apocrine metaplasia were selected following histologic examination. 
Twenty cases were benign (7 apocrine cysts with some degree of papillary hyperplasia, 7 apocrine adenosis, 3 intraductal papillomas, 2 ductal adenomas, and 1 adenomyoepithelioma), 1 case was considered borderline (atypical apocrine adenosis [5])), and 22 cases were diagnosed as malignant (9 intraductal carcinomas of apocrine type and 13 invasive apocrine carcinomas).

The definition of apocrine metaplasia was based on the criteria reported by O'Malley et al. (3) and Tavassoli and Norris (2). It is summarized as follows: (1) markedly eosinophilic cytoplasm with fine granularity, (2) large and moderately vesicular nuclei with an occasional prominent red nucleoli, and (3) the occasional presence of apical snouts. A potential marker for apocrine differentiation, gross cystic disease fluid protein-15 (GCDFP-15), was consistently positive in all of the cases examined in this study. However, the presence of GCDFP-15 alone was not considered as apocrine metaplasia because of the reported presence of GCDFP-15 immunoreactivity in nonapocrine breast epithelial cells (9), and, therefore, we did not include this in the criteria in defining apocrine metaplasia in our study.

One representative block from each case was examined in this study. Sections of 3 to $4 \mu \mathrm{M}$ were dehydrated and rehydrated in graded alcohol. The sections were then placed in a pressure cooker in a closed plastic container filled with $10 \mathrm{~mm}$ citric acid ( $\mathrm{pH}$ 6.0) for $10 \mathrm{~min}$ to do epitope retrieval, and then endogenous peroxide was blocked with $0.3 \%$ hydrogen peroxidase for $10 \mathrm{~min}$. The primary antibodies used were anti-Ki-67 (MIB-1, mouse monoclonal, dilution 1:100, $60 \mathrm{~min}$; Immunotech, Inc., Westbrook, ME), anti-p53 (CM1, rabbit polyclonal, dilution 1:600, $30 \mathrm{~min}$; Novocastra Laboratories, Newcastle upon Tyne, UK), anti-p21 (clone Cip1, mouse monoclonal, dilution 1:500, $30 \mathrm{~min}$; Santa Cruz Biochemistry, Santa Cruz, CA), and anti-p27 (clone Kip1, mouse monoclonal, dilution 1:500, 30 min; Transduction Laboratory, Lexington, KY). These primary antibodies were incubated at room temperature in a moist chamber. Immunostaining was performed by Envision system (DAKO Japan, Kyoto, Japan), and the peroxidase reaction was developed using diaminobenzidine. A light Mayer's hematoxylin was used as a counterstain.

Negative controls were performed using $0.01 \mathrm{M}$ phosphate buffered saline instead of the primary antibodies above. No specific immunoreactivity was detected in these negative control sections. Only nuclear immunoreactivity was regarded as positive. A total of 500 cells were examined, and the cases that demonstrated more than $10 \%$ (50 cells) positive cells were determined to be positive cases. Statistical analysis was determined by either $\chi^{2}$ test or two sample $t$ tests.

\section{RESULTS}

\section{Histopathologic Findings}

Seven cases of benign apocrine cysts were associated with papillary epithelial hyperplasia (Fig. 1). Stratification of apocrine metaplastic cells without intervening fibrovascular stroma was detected in these benign cases. Nuclei were usually small and uniform. Apocrine adenosis was usually associated with some degree of nuclear enlargement. However, the lobulocentricity of the lesion, as well as the presence of a myoepithelial layer, excluded the possibility of invasive carcinoma. Apocrine metaplasia was seen focally in the lesions of intraductal papillomas, ductal adenomas, or adenomyoepithelioma, without severe nuclear atypia. Borderline lesions with uncertain malignant potential and atypical apocrine adenosis were detected in one case. A 3 -fold variation in nuclear size was detected focally in these cases (Fig. 2).

All nine intraductal carcinomas with diffuse apocrine cytology were associated with moderate to severe degrees of nuclear atypia. Nuclear enlargement, variation in nuclear size, or presence of macronucleoli was detected in these cases. Architecturally, all nine cases demonstrated cribriform, micropapillary, or papillary patterns (Fig. 3). Tumor cells were evenly distributed, and sharply defined glandular or luminal spaces and/or rigid papillae were detected in all of these cases. Five of nine cases demonstrated the presence of comedonecrosis. We could not find any cases of extremely lowgrade apocrine intraductal carcinoma or atypical ductal hyperplasia of apocrine type. One case demonstrated architecture similar to that of atypical apocrine adenosis, but the presence of the macronucleoli, as well as nuclear pleomorphism, confirmed the diagnosis of intraductal apocrine carcinoma in sclerosing adenosis (Fig. 4). Invasive

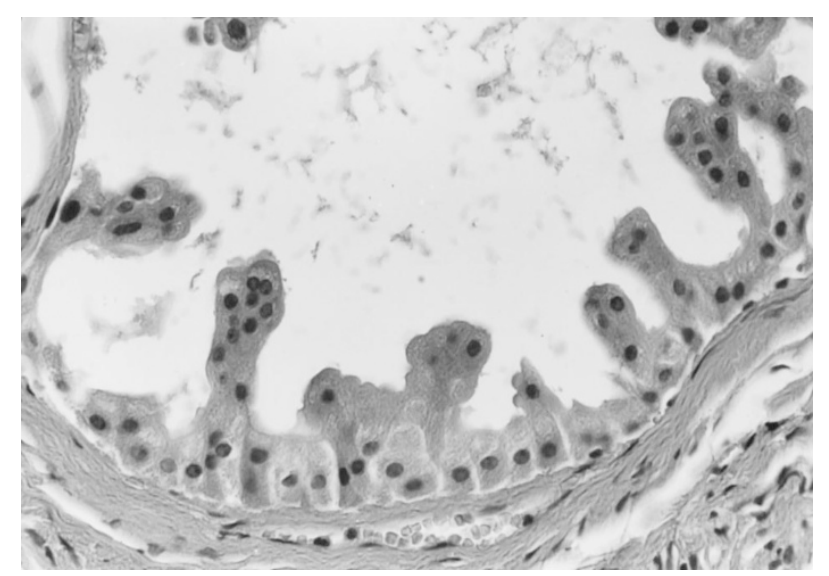

FIGURE 1. Benign apocrine cyst with papillary epithelial hyperplasia. Stratification of apocrine metaplastic cells without intervening fibrovascular stroma were seen (hematoxylin and eosin stain; original magnification, $200 \times$ ). 


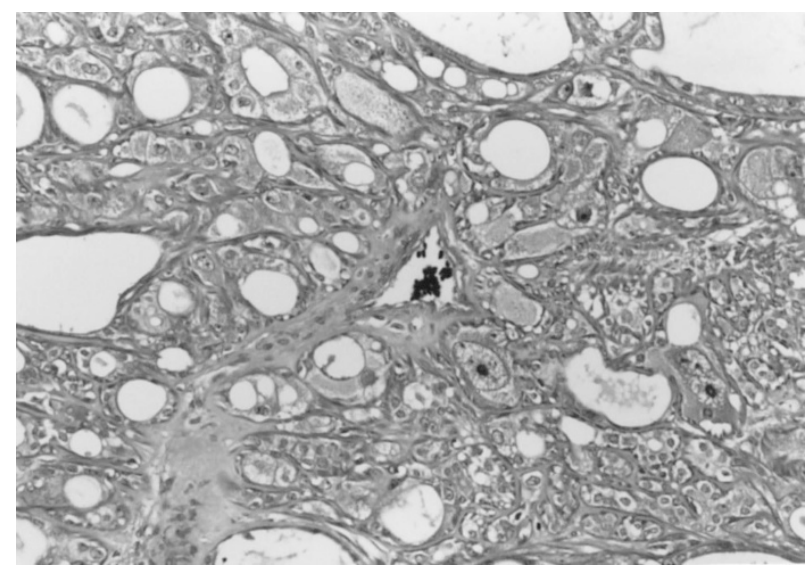

FIGURE 2. Atypical apocrine adenosis. A 3-fold variation in nuclear size was detected focally (hematoxylin and eosin stain; original magnification, $200 \times$ ).

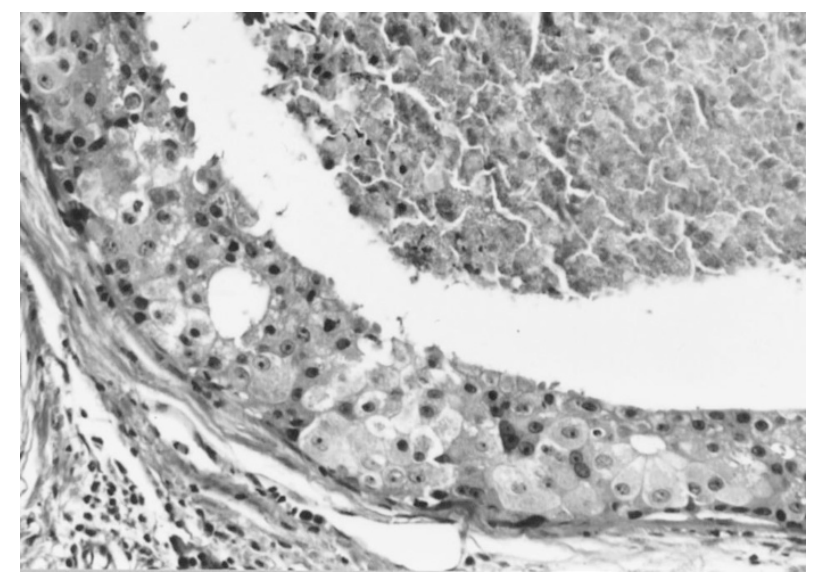

FIGURE 3. Intraductal apocrine carcinoma. Atypical apocrine epithelium with cribriform structure and comedonecrosis (hematoxylin and eosin stain; original magnification, $200 \times$ ).

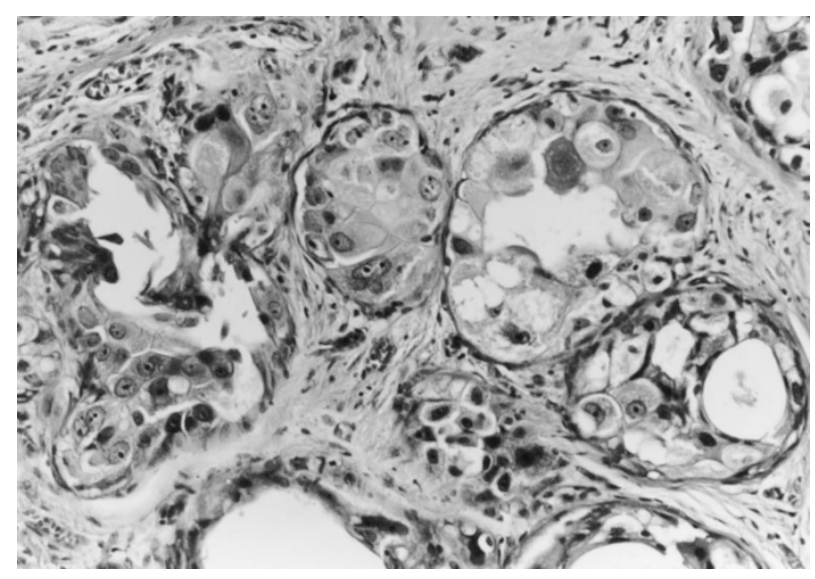

FIGURE 4. Intraductal apocrine carcinoma in adenosis. The presence of macronucleoli as well as nuclear pleomorphism were diffusely distributed (hematoxylin and eosin stain; original magnification, 200×).

apocrine carcinoma (Fig. 5) had variable architectural patterns, including papillotubular, solid, or trabecular. Nuclear grade was also moderate to severe.

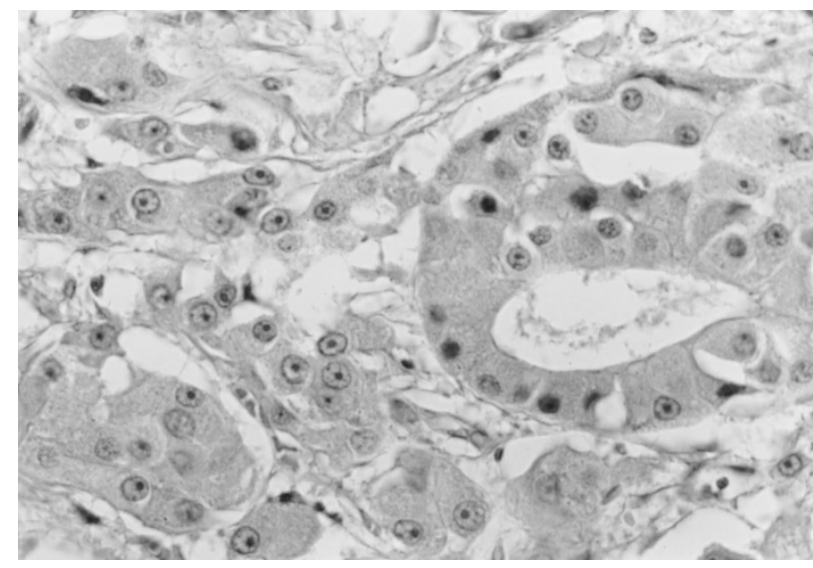

FIGURE 5. Invasive apocrine carcinoma. Nuclear grade is moderate to severe (hematoxylin and eosin stain; original magnification, $400 \times$ ).

\section{Immunohistochemistry}

Results are summarized in Tables 1 and 2. Only one case of intraductal papilloma with focal apocrine metaplasia was positive for Ki-67 or MIB-1 immunoreactive protein ( $11 \%$ of the apocrine epithelial cells were positive) among benign cases. A borderline malignant case (atypical apocrine adenosis) was also negative. The ratio of positive cases in malignant lesions (Fig. 6) (17 of 21 [88.9\%]) was significantly higher than that of benign lesions (1 of $19[5.3 \%])(P<.001)$. None of the benign or borderline cases was positive for p53 immunoreactivity, and 15 of 22 malignant cases (68.2\%) were positive for p53 $(P<.001)$. All nine intraductal carcinomas were positive for p53 (100\%) (Fig. 7). The rate of immunoreactive p53 protein in intraductal carcinoma was statistically more frequent than that of invasive carcinomas $(46.2 \% ; P<.01)$. The rate of p21 immunoreactivity in malignant cases (14 of 22 [63.6\%]) was slightly higher than in benign cases (7 of 19 [36.8\%]), but the difference did not reach statistical significance.

In malignant cases, the mean percentage of Ki67 -positive cells or Ki-67 labeling index was $15.0 \%$, which was significantly higher than in benign cases (average, 2.7\%; $P<$.005). All benign or borderline cases were negative for $\mathrm{p} 53$, whereas all but one case among the malignant cases were positive for p53. The mean percentage of p53-positive cells was $23.4 \%$ in carcinoma cases. Intraductal carcinomas demonstrated a higher percentage of p53-positive ratio than invasive carcinomas, but the differences did not reach statistical significance. The mean percentage of p27-positive cells was slightly higher in malignant $(23.5 \%)$ than in benign cases $(7.9 \%)$, but the differences did not reach statistical significance.

Among carcinoma cases, those with high nuclear grade (NG3: 13 cases, 6 intraductal and 7 invasive ductal) were compared with those with intermediate nuclear grade (NG2: 9 cases, 3 intraductal and 6 
TABLE 1. Summary of Results of Benign, Borderline, and Malignant Apocrine Lesions Showing Positive Immunoreactivity for Ki-67, p53, p21, and p27 (Positive Cells with More Than 10\% Staining)

\begin{tabular}{|c|c|c|c|c|}
\hline & Ki-67 & p53 & $\mathrm{p} 21$ & p27 \\
\hline Benign $(n=20)$ & $1 / 19(5.3 \%)^{a}$ & $0 / 20(0.0 \%)^{a}$ & $7 / 19$ (36.8\%) & $12 / 18(66.7 \%)$ \\
\hline Borderline $(n=1)$ & $0 / 1(0 \%)$ & $0 / 1(0 \%)$ & $0 / 1(0 \%)$ & $1 / 1(100 \%)$ \\
\hline Malignant $(n=22)$ & $17 / 21(81.0 \%)^{a}$ & $15 / 22(68.2 \%)^{a}$ & $14 / 22(63.6 \%)$ & $11 / 21(52.4 \%)$ \\
\hline DCIS $(n=9)$ & $8 / 9(88.9 \%)$ & $9 / 9(100 \%)^{b}$ & $6 / 9(66.7 \%)$ & $6 / 9(66.7 \%)$ \\
\hline IDC $(n=13)$ & $9 / 12(75.0 \%)$ & $6 / 13(46.2 \%)^{b}$ & $8 / 13(61.5 \%)$ & $5 / 12(41.7 \%)$ \\
\hline
\end{tabular}

DCIS, intraductal carcinoma, apocrine type; IDC, invasive apocrine carcinoma.

${ }^{a} p<.001$

${ }^{b} p<.01$.

TABLE 2. Summary of Results Showing the Percentage of Positive Immunoreactive Cells for Ki-67, p53, p21, and p27 in Benign, Borderline, and Malignant Apocrine Lesions

\begin{tabular}{lcccc}
\hline & Ki-67 & p53 & p21 & p27 \\
\hline Benign $(\mathrm{n}=20)$ & $2.7 \pm 2.9^{a}$ & $0.0 \pm 0.0^{a}$ & $7.9 \pm 8.5$ & $15.8 \pm 9.4$ \\
Borderline $(\mathrm{n}=1)$ & 3 & 0 & 0 & 26 \\
Malignant $(\mathrm{n}=22)$ & $15.0 \pm 6.4^{a}$ & $23.4 \pm 19.6^{a}$ & $23.5 \pm 17.6$ & $14.9 \pm 12.2$ \\
DCIS $(\mathrm{n}=9)$ & $14.7 \pm 3.9$ & $37.4 \pm 18.0$ & $28.0 \pm 21.6$ & $19.4 \pm 13.4$ \\
IDC $(\mathrm{n}=13)$ & $15.3 \pm 8.4$ & $13.7 \pm 12.9$ & $20.3 \pm 14.4$ & $11.4 \pm 10.5$ \\
\hline
\end{tabular}

DCIS, intraductal carcinoma, apocrine type; IDC, invasive apocrine carcinoma.

Mean $\pm \mathrm{SD}$

${ }^{a} p<.005$.

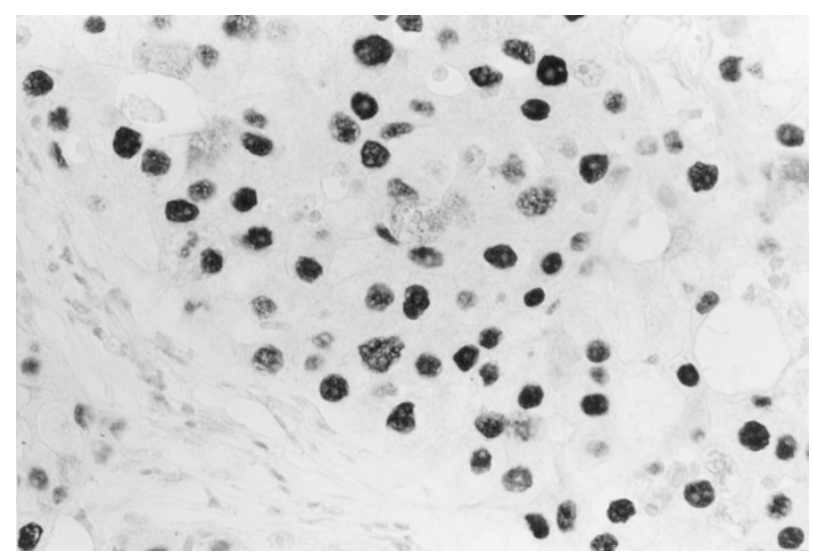

FIGURE 6. Strong nuclear reactivity in Ki-67 or MIB-1. Invasive apocrine carcinoma (original magnification, $400 \times$ ).

invasive). The ratio of Ki-67-positive cases (NG3: 11 of 12; NG2: 6 of 9) and the percentage of Ki-67positive cells (NG3: 15.0\%; NG2: $15.1 \%$ ) were not different between these two groups of the carcinoma cases. The ratio of p53-positive cases was significantly higher in NG3 cases (11 of 13 [84.6\%]) than in NG2 cases (4 of 9 [44.4\%]; $P<.05$ ). The percentage of p53-positive cells in NG3 cases $(30.5 \%)$ was higher than in NG2 cases $(13.2 \%)$, but it was not statistically significant.

No other significant differences in the immunohistochemical localization were detected among different types of benign lesions, subtypes of intraductal carcinomas (presence of comedonecrosis, nuclear grade, structural subtype), or the histologic grade of invasive carcinomas (tubule formation, nuclear pleomorphism, mitotic counts).

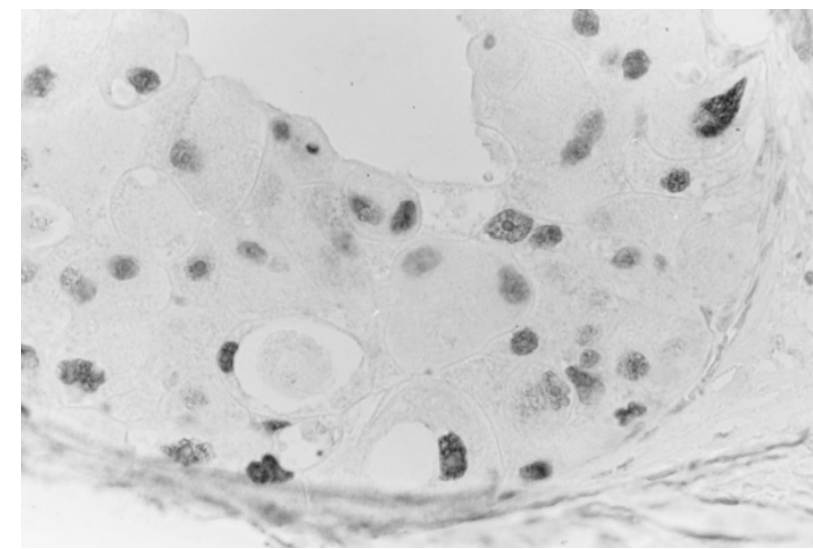

FIGURE 7. Positive cases for p53 in intraductal apocrine carcinoma (original magnification, $400 \times$ ).

\section{DISCUSSION}

It is occasionally very difficult to determine benignity or malignancy in proliferative breast disorders with diffuse apocrine differentiation or metaplasia $(3,8)$. Abati et al. (10) reported that the most consistent and important cytologic feature in apocrine carcinoma is high nuclear grade in a great majority of tumor cells and prominent eosinophilic nucleoli. The cytologic features described by Abati et al. are also consistent with our study. However, results from our study demonstrated that immunolocalization of Ki-67 and p53 may contribute to the differential diagnosis of breast lesions with diffuse apocrine metaplasia. Both the number of positive cases and the percentage of positive tumor cells were significantly higher in malignant apocrine cases than in benign apocrine cases. There was only one case of benign lesions with more than $10 \%$ Ki-67 positivity. None of the benign or borderline cases examined in our study was associated with p53 immunoreactivity. These results suggest that the Ki-67 and/or p53 immunohistochemistry can be an auxiliary method for determining the possible biologic behavior of lesions, as reported in other nonapocrine breast ductal proliferative disorders $(11,12)$.

Ki-67 labeling index was higher in carcinoma than in atypical ductal hyperplasia (13) and higher in carcinoma than in normal breast (11). P53 im- 
munoreactivity was generally not detected immunohistochemically before the carcinoma in situ phase throughout the course of possible breast carcinoma development (12). Results from our study revealed that there was a difference in the patterns of p53 immunoreactivity between apocrine and nonapocrine carcinomas. In apocrine carcinoma, p53 immunoreactivity in intraductal carcinoma was more frequently positive than in invasive ductal carcinoma, and high nuclear grade carcinoma was more frequently positive than intermediate nuclear grade carcinoma. Six of 9 intraductal carcinomas $(66.7 \%)$ were of high nuclear grade, and 5 had extensive comedonecrosis, whereas 7 of 13 invasive apocrine carcinomas (53.8\%) were of high nuclear grade. P53 immunoreactivity was reported to be strongly correlated with nuclear grade in both intraductal (14) and invasive ductal nonapocrine carcinomas (15). Therefore, the roles of p53 in carcinoma development may be different between apocrine and nonapocrine carcinomas, but it awaits further investigations for clarification. These results also suggest that both Ki-67 and p53 immunostaining can be good markers for differentiating malignant apocrine lesions.

When matched for age, in general, the relative risk for developing subsequent invasive carcinoma for atypical ductal hyperplasia falls between benign proliferative changes and in situ carcinomas (16). Conversely, the classification of borderline lesions with diffuse apocrine cytology, which may have a similar spectrum of lesions, has not been universally accepted, and the biologic behavior of borderline lesions is not well established. Borderline apocrine lesions are classified into two categories (5, 17): atypical apocrine adenosis and atypical ductal hyperplasia of apocrine type $(2,7)$. Atypical apocrine adenosis represents the lesion of adenosis with diffuse apocrine cytology and some degree of nuclear atypia. The enlarged nucleoli and greater than 3-fold variation in nuclear area may be required (17). Two cases in our study initially had been considered to meet the criteria for this category. However, one case demonstrated only focal nuclear pleomorphism and was reclassified into benign lesions. Neither Ki-67 nor p53 was positive in these cases. Another case was diagnosed as intraductal apocrine carcinoma within the sclerosing adenosis because of the prominence of macronucleoli and diffuse nuclear pleomorphism. The cases of atypical ductal hyperplasia of apocrine differentiation were not available for our present study.

In a study on intraductal apocrine carcinoma by Tavassoli and Norris (2), 32 of 37 cases (86.5\%) were of high grade, based on cytologic atypia and intraluminal necrosis. The remaining five cases were of intermediate grade. Similarly, the current study has demonstrated that six of nine intraductal apocrine carcinomas were high grade, and the remaining three were intermediate grade. Therefore, the borderline intraductal apocrine lesions, atypical hyperplasia to low-grade intraductal carcinoma, may in fact be rare. Further studies for collecting borderline malignancy and low-grade carcinoma cases are required to clarify further biologic differences between benign and malignant apocrine lesions.

Finally, p21 and p27 immunoreactive proteins were not effective for the apocrine lesions investigated in this study. In breast carcinoma cases, an inverse expression of $\mathrm{p} 21$ and p53 proteins has been reported (18). Reduced immunohistochemical expression of p27 in breast carcinoma in comparison to benign lesions has also been reported (19). Low nuclear expression of p27 within cancer cells may suggest poor survival for the patient (19). Our results showed that apocrine lesions, regardless of their biologic behavior, tend to be stained far less than in $50 \%$ of the population. However, further studies are required for clarification.

In conclusion, our study may have very important clinicopathologic implications in the diagnosis and prognosis of patients with apocrine lesions of the breast.

Acknowledgment: The authors thank the staff at the Department of Pathology, Kawasaki Hospital, Kawasaki Medical School, and Ms. Masako Uehira for their technical assistance. We thank Dr. Kojiro Shimozuma, Kawasaki Medical School, Kurashiki, Japan; Dr. Mamoru Mochizuki, Jhoban Hospital, Iwaki, Japan; Dr. Yojiro Orita and Dr. Shigeo Shiiki, Chugoku Central Hospital, Fukuyama, Japan; Dr. Miki Harada, Kanto Teishin Hospital, Tokyo, Japan; Dr. Yasuo Nakata, Hyogo Medical School, Nishinomiya, Japan; Dr. Suzuko Moritani, Shiga Medical School, Otsu, Japan; Dr. Toshiharu Matsumoto, Juntendo University School of Medicine, Tokyo, Japan; Dr. Teiichi Motoyama, Yamagata University School of Medicine, Yamagata, Japan; Dr. Ryoichi Kurano, Kumamoto Ishikai Kumamotochiki Medical Center, Kumamoto, Japan; Dr. Hiroshi Kudoh, Tottori Red Cross Hospital, Tottori, Japan; Dr. Shu Nakamoto, Tottori Prefectural Central Hospital, Tottori, Japan; Dr. Kenji Notohara, Okayama University School of Medicine, Okayama, Japan; Dr. Kuniyasu Okazaki, Kunitomi Clinic, Okayama, Japan; and Dr. Kazuo Nakanishi, Iizuka Hospital, Iizuka, Japan, for allowing us to examine and study cases from their hospitals. In addition, we thank Mr. Andrew Darnel for his kind editing of this manuscript.

This work is supported in part by the grant-in-aid for cancer research 7-1 from the Ministry of Health and Welfare, Japan, a grant-in-aid for scientific re- 
search on priority area (A-11137301) from The Ministry of Education, Science and Culture, Japan, a grant-in-aid for scientific research (B-11470047) from Japan Society for the Promotion of Science, and a grant from The Naitou Foundation and Suzukenn Memorial Foundation.

\section{REFERENCES}

1. Moriya T, Silverberg SG. Intraductal carcinoma of the breast. Int J Surg Pathol 1995;3:83-92.

2. Tavassoli FA, Norris HJ. Intraductal apocrine carcinoma: a clinicopathologic study of 37 cases. Mod Pathol 1994; 7:813-8.

3. O'Malley FP, Page DL, Nelson EH, Dupont WD. Ductal carcinoma in situ of the breast with apocrine cytology: definition of a borderline category. Hum Pathol 1994;25:164-8.

4. Simpson JF, Page DL, Dupont WD. Apocrine adenosis: a mimic of mammary carcinoma. Surg Pathol 1990;3:289-99.

5. Carter DL, Rosen PP. Atypical apocrine metaplasia in sclerosing lesions of the breast: a study of 51 patients. Mod Pathol 1991;4:1-5.

6. Page DL, Dupont WD, Jensen RA. Papillary apocrine change of the breast: associations with atypical hyperplasia and risk of breast cancer. Cancer Epidemiol Biomarkers Prev 1996;5: 29-32.

7. Lininger RA, Tavassoli FA. Atypical intraductal hyperplasia of the breast. In: Silverstein MJ, editor. Ductal carcinoma in situ of the breast. Baltimore: Williams \& Wilkins; 1997. pp. 195-222.

8. Silverberg SG, Masood S. The breast. In: Silverberg SG, Delellis RA, Frable WJ, editors. Principles and practice of surgical pathology and cytopathology. 3rd ed. New York: Churchill Livingstone; 1997. pp. 575-674.

9. Losi L, Lorenzini R, Eusebi V, Bussolati G. Apocrine differentiation in invasive carcinoma of the breast: comparison of monoclonal and polyclonal gross cystic disease fluid protein-15 antibodies with prolactin-inducible protein
mRNA gene expression. Appl Immunohistochem 1995; 3:91-8.

10. Abati AD, Kimmel M, Rosen PP. Apocrine mammary carcinoma: a clinicopathologic study of 72 cases. Am J Clin Pathol 1990;94:371-7.

11. Pavelic ZP, Pavelic L, Lower EE, Gapany M, Gapany S, Barker EA, et al. C-myc, c-erbB-2, and Ki-67 expression in normal breast tissue and in invasive and noninvasive breast carcinoma. Cancer Res 1992;52:2597-602.

12. Eriksson ET, Schimmelpenning H, Aspenblad U, Zetterberg A, Auer GU. Immunohistochemical expression of the mutant p53 protein and nuclear DNA content during the transition from benign to malignant breast disease. Hum Pathol 1994; 25:1228-33.

13. Hoshi K, Tokunaga M, Mochizuki M, Ohtake T, Katagata N, Wakasa $\mathrm{H}$, et al. Pathological characterization of atypical ductal hyperplasia of the breast (Japanese). Gan to Kagaku Ryoho 1995;22:S36-S41.

14. O'Malley FP, Vnencak-Jones CL, Dupont WD, Parl F, Manning S, Page DL. P53 mutations are confined to the comedo type ductal carcinoma in situ of the breast: immunohistochemical and sequencing data. Lab Invest 1994;71:67-72.

15. Rosen PP, Lesser ML, Arroyo CD, Cranor M, Borgen P, Norton L. P53 in node-negative breast carcinoma: an immunohistochemical study of epidemiologic risk factors, histologic features and prognosis. J Clin Oncol 1995;13:821-30.

16. Page DL, Dupont WD, Rogers LW, Rados MS. Atypical hyperplastic lesion of the breast: a long-term follow-up study. Cancer 1985;55:2698-708.

17. Seidman JD, Ashton M, Lefkowitz M. Atypical apocrine adenosis of the breast: a clinicopathologic study of 37 patients with 8.7-year follow-up. Cancer 1996;77:2529-37.

18. Wakasugi E, Kobayashi T, Tamaki Y, Ito Y, Miyashiro I, Komoike Y, et al. P21(Waf1/Kip1) and p53 protein expression in breast carcinoma. Am J Clin Pathol 1997;107:684-91.

19. Catzavelos C, Bhattacharya N, Ung YC, Wilson JA, Roncari L, Sandhu C, et al. Decreased levels of the cell-cycle inhibitor p27Kip1 protein: prognostic implications in primary breast cancer. Nat Med 1997;3:227-30. 